Complex windmill transformation producing new purely magnetic fluids

This article has been downloaded from IOPscience. Please scroll down to see the full text article.

2011 Class. Quantum Grav. 28075015

(http://iopscience.iop.org/0264-9381/28/7/075015)

View the table of contents for this issue, or go to the journal homepage for more

Download details:

IP Address: 131.211.213.168

The article was downloaded on 15/02/2013 at 08:14

Please note that terms and conditions apply. 


\title{
Complex windmill transformation producing new purely magnetic fluids
}

\author{
C Lozanovski ${ }^{1}$ and $\mathbf{L}$ Wylleman ${ }^{2,3}$ \\ ${ }^{1}$ Computational Engineering and Science Research Centre (CESRC) and The Faculty of \\ Education, University of Southern Queensland, Springfield Queensland 4300, Australia \\ ${ }^{2}$ Department of Mathematics, Utrecht University, 3584 CD Utrecht (Budapestlaan 6), \\ The Netherlands \\ ${ }^{3}$ Department of Mathematical Analysis, Ghent University, B - 9000 Ghent, Belgium \\ E-mail: con.lozanovski@usq.edu.au and Lode.Wylleman@UGent.be
}

Received 14 September 2010, in final form 9 February 2011

Published 10 March 2011

Online at stacks.iop.org/CQG/28/075015

\begin{abstract}
Minimal complex windmill transformations of $G_{2} I \mathrm{~B}$ (ii) spacetimes (admitting a two-dimensional Abelian group of motions of the so-called Wainwright B(ii) class) are defined and the compatibility with a purely magnetic Weyl tensor is investigated. It is shown that the transformed spacetimes cannot be perfect fluids or purely magnetic Einstein spaces. We then determine which purely magnetic perfect fluids (PMpfs) can be windmill-transformed into purely magnetic anisotropic fluids (PMafs). Assuming separation of variables, complete integration produces two, algebraically general, $G_{2} I$-B(ii) PMpfs: a solution with zero 4-acceleration vector and spatial energy-density gradient, previously found by the authors, and a new solution in terms of Kummer's functions, where these vectors are aligned and non-zero. The associated windmill PMafs are rotating but non-expanding. Finally, an attempt to relate the spacetimes to each other by a simple procedure leads to a $G_{2} I$-B(ii) oneparameter PMaf generalization of the previously found metric.
\end{abstract}

PACS numbers: 04.20.-q, 02.40.Tt, 04.20.Jb

\section{Introduction}

Complex transformations have long been used to either relate two known (real) spacetimes or generate a new (real) spacetime from a known one, see [1] for various discussions, and have been successfully applied to higher-dimensional spacetimes [2]. Formally, there has been extensive investigations [3-9] of complex relativity with the aim of obtaining real spacetimes as real slices of complex spacetimes (also see parts of [10] for a brief but concise review). 
In addition, the complex point of view furnishes a way of classifying real spacetimes and is therefore of considerable interest.

A fact exploited in this study is that properties of the kinematic quantities, in general, are not preserved under the action of complex transformations. This extends to the form of the Ricci tensor and Petrov type of the Weyl tensor. However, there are cases in which either the form of the Ricci tensor or Petrov type (or both) are preserved. An example of a kinematic non-preserving but Ricci and Weyl (Petrov type) preserving complex transformation is the original Newman-Janis transformation ${ }^{4}$ of Schwarzschild to Kerr [11] (for more details see [12-14], also [15] for extension to Kerr-Newman and [16-21] for other applications). It is interesting to note that while the Petrov type is preserved in this case, the nature of the gravito-electromagnetic tensors are mapped from purely electric (Schwarzschild) to the general case (Kerr) - neither purely electric nor purely magnetic. The same holds for the complex transformation of Kasner to vacuum windmill ${ }^{5}$ (i.e the Lewis spacetime [23] or, in the notation of McIntosh, vacuum 'windmill' spacetime) [22]. However, preserving the nature of the gravito-electromagnetic tensors, in addition to the form of the Ricci, is also possible, as demonstrated by the well-known relationship between the Weyl-W2 [1, 24] and Kasner $[25,26]$ spacetimes. Note that preserving the nature of the gravito-electromagnetic tensors, even in the purely electric or purely magnetic case, is not sufficient to preserve the Petrov type.

Preserving the purely electric or purely magnetic condition under the action of complex transformations is an intriguing possibility. Especially from the perspective of generating new solutions. This is of particular interest in the purely magnetic case as there are relatively few known examples compared to the purely electric case. Moreover, there appears to be a lack of specific generating procedures, apart from simply conformally relating a purely magnetic spacetime. These key ideas and requirements form the main motivation of this investigation.

More specifically, the suggestion by McIntosh [22] to further investigate complex transformations of Wainwright [27] class B(ii) (orthogonally transitive $G_{2} I$ acting on a $S_{2}$ with two hypersurface orthogonal Killing vectors (HOKV)) spacetimes,

$$
g=\exp (2 b) \mathrm{d} x^{2}+\exp (h)\left[\exp (2 k) \mathrm{d} y^{2}+\exp (-2 k) \mathrm{d} z^{2}\right]-\exp (2 a) \mathrm{d} w^{2},
$$

is considered in the non-vacuum case under a specific set of assumptions. Note that separable perfect fluid spacetimes of this class have attracted considerable attention and the general solution is known; stiff-matter [28], linear equation of state and conformally-flat spatial 3slices (for the latter, see Wainwright [29]) [30], general case [31] and for non-comoving, general properties and solutions, see [32, 33].

For future reference, these transformations are referred to as complex windmill transformations or minimal complex windmill transformations if the related spacetimes share all metric coefficients (see sub-section 2.1). Under the action of this transformation it will be assumed that the purely magnetic condition is preserved while the energy-momentum tensor is transformed from a perfect to anisotropic fluid. Moreover, this class of PMpf spacetimes considered here is general enough to possibly include members where the spatial gradient of the energy-density function and 4-acceleration vector are both non-zero. Kinematically, one of the appealing consequences of complex windmill transformations is that, in general, irrotational spacetimes map to rotating spacetimes. This is of importance as there are very few known examples of rotating purely magnetic spacetimes.

\footnotetext{
4 Commonly referred to as the Newman-Janis algorithm (NJA).

5 The term, vacuum 'windmill' spacetime, was coined by McIntosh [22] to describe the transitional nature of the light-cone as measured by an observer moving along a spatial axis. We shall adopt the word 'windmill' to distinguish our definition of the complex transformation under consideration.
} 
One of the main theorems of the Wylleman-Van den Bergh [34] classification of irrotational and non-accelerating, type I, PMpf spacetimes dictates that if any two of the following conditions hold (which imply the third):

(1) the fluid 4-velocity is irrotational;

(2) the fluid 4-velocity is non-accelerating (i.e. the spatial gradient of the pressure function vanishes);

(3) the spatial gradient of the energy-density function vanishes;

then (up to a constant re-scaling of the metric) there exists a unique PMpf spacetime ${ }^{6}$. More broadly, the classification includes the possible entry of irrotational PMpf spacetimes where the spatial gradient of the energy-density function and 4-acceleration vector are both non-zero. This possibility was indicated with a question mark as it was unknown if this class is empty or non-empty (see figure 1 of [34]). It is shown here that this class is non-empty by producing an explicit example.

In section 2, a general complex windmill transformation of $G_{2} I-\mathrm{B}$ (ii) spacetimes is defined and the form of the resulting windmill spacetime is presented. The concept of a minimal complex windmill transformation is defined and the assumptions on the energy-momentum and Weyl tensors are stated. The general forms of the energy-momentum tensors are defined and the kinematic properties are calculated for each spacetime. In section 3 , it is shown that windmill perfect fluids or purely magnetic Einstein spaces do not exist, while in section 4 $G_{2} I$-B(ii) PMpf spacetimes are considered. It is shown that there are two PMpf spacetimes that have related windmill PMaf spacetimes. One of these is the PMpf solution independently found by the present authors [34] (henceforth, referred to as the WVL spacetime), and the other is given by a general solution to the confluent hypergeometric equation by Kummer's functions. The spatial gradient of the energy-density function and the 4-acceleration vector are both 0 for the former spacetime while non-zero for the latter. In section 5 , an attempt to relate these spacetimes to each other by interchanging coordinates of the metric coefficients and introducing an appropriate conformal factor leads to a one parameter PMaf generalization of the WVL PMpf spacetime.

\section{Complex windmill transformation}

The concept of a complex 'windmill' transformation is a straightforward generalization of the type of transformation McIntosh [22] used to obtain a vacuum 'windmill' spacetime. In this section a concise definition of a complex windmill transformation is given, as are the assumptions made on the properties of the related spaetimes and the kinematic properties of these spacetimes.

\section{1. $G_{2} I-B(i i)$ and related windmill spacetime}

A general complex windmill transformation of $(1), \mathcal{C}(g)=\hat{g}$, is defined by

$$
\mathcal{C}:\{a, b, h, k ; y, z\} \mapsto\left\{\hat{a}, \hat{b}, \hat{h}, \mathrm{i} \hat{k} ; \frac{1}{\sqrt{2}}(u+\mathrm{i} v), \frac{1}{\sqrt{2}}(u-\mathrm{i} v)\right\}
$$

and leads to a windmill spacetime of the MacCallum-McIntosh form [36]:

$$
\hat{g}=\epsilon_{1} \exp (2 \hat{a}) \mathrm{d} w^{2}+\epsilon_{2} \exp (2 \hat{b}) \mathrm{d} x^{2}+\epsilon_{3} \exp (\hat{h})\left[\cos (2 \hat{k})\left(\mathrm{d} u^{2}-\mathrm{d} v^{2}\right)-2 \sin (2 \hat{k}) \mathrm{d} u \mathrm{~d} v\right]
$$

${ }^{6}$ It should be emphasized that this is a uniqueness theorem. Therefore, investigations assuming any of the two conditions above will lead to the WVL spacetime. This is the case for the purely magnetic non-tilted Bianchi-type $V I_{0}$ cosmological models considered in [35]. This will be commented on further elsewhere. 
where $\{\hat{a}, \hat{b}, \hat{h}, \hat{k}\}$ are the real functions of $w$ and $x$ only, and the signature is preserved by the set of constants $\epsilon_{1}, \epsilon_{2}, \epsilon_{3} \in\{-1,+1\}$, see appendix A. It is interesting to note that MacCallum [36] has shown that orthogonally transitive $G_{2} I$ spacetimes (in the form (4) of [36]) admit a HOKV (neither $\xi^{a}=(0,0,1,0)$ nor $\eta^{a}=(0,0,0,1)$ but a linear combination of these) iff certain metric coefficients satisfy a linear relation. It follows that if there is one HOKV then there must be a second orthogonal to the first [37] and if they constitute a pair of complex conjugate HOKVs then the spacetime is of the form (3).

Note that if a complex windmill transformation leads to a complex spacetime, i.e. the requirement $k \mapsto \mathrm{i} \hat{k} \Rightarrow\{\hat{a}, \hat{b}, \hat{h}, \hat{k}\} \in \mathbb{C}$ (in general), then such spacetimes are not considered further. On the other hand, if the entire transformation itself turns out to be real then this is considered trivial. A trivial complex windmill transformation will be defined below, in subsection 2.3, in terms of kinematic quantities.

For simplicity a special case of (2) will be considered in this study. It is assumed that spacetime (3) inherits the metric coefficients of (1) in the following sense.

Definition 2.1. A minimal complex windmill transformation of (1) is defined by

$$
\mathcal{C}^{\circ}:\{a, b, h, k ; y, z\} \mapsto\left\{a, b, h, i k ; \frac{1}{\sqrt{2}}(u+i v), \frac{1}{\sqrt{2}}(u-i v)\right\} .
$$

\subsection{Energy-momentum and weyl tensors}

In this study the following assumptions are made.

\section{Assumptions 2.1.}

(1) The energy-momentum tensor of any one of the spacetimes (1) or (3) is in the form of a perfect fluid while the other an anisotropic fluid (which may degenerate to a perfect fluid).

(2) The minimal complex windmill transformation preserves the purely magnetic property.

(3) The $G_{2} I-B(i i)$ and windmill spacetimes are assumed not to be both conformally flat.

Specifically, for (1),

$$
\left\{\exists u^{a}: u_{a} u^{a}=-1, E_{a c}=0, \operatorname{ein}^{(\mathrm{af})}{ }_{a c} \equiv R_{a c}-\frac{1}{2} R g_{a c}-T_{a c}^{(\mathrm{af})}=0\right\}
$$

where, see for example Maartens et al [38],

$$
T_{a c}^{(\text {af })}=\left(\rho+p_{\perp}\right) u_{a} u_{c}+p_{\perp} g_{a c}+\left(p_{\|}-p_{\perp}\right) s_{a} s_{c},
$$

and the fluid 4-velocity, $u^{a}$, satisfies $u_{a} s^{a}=0$ and $s_{a} s^{a}=1$. Further, $\rho$ is the energy-density function while $p_{\perp}$ and $p_{\|}$denote the pressure functions perpendicular and parallel to $s^{a}$, respectively. On the other hand, for (3), which is related to (1) by (4),

$$
\left\{\exists \hat{u}^{a}: \hat{u}_{a} \hat{u}^{a}=-1, \hat{E}_{a c}=0, \quad \text { ein }{ }^{(\hat{\mathrm{a}})}{ }_{a c} \equiv \hat{R}_{a c}-\frac{1}{2} \hat{R} \hat{g}_{a c}-\hat{T}_{a c}^{(\mathrm{af})}=0\right\}
$$

where

$$
\hat{T}_{a c}^{(\text {af })}=\left(\hat{\rho}+\hat{p}_{\perp}\right) \hat{u}_{a} \hat{u}_{c}+\hat{p}_{\perp} \hat{g}_{a c}+\left(\hat{p}_{\|}-\hat{p}_{\perp}\right) \hat{s}_{a} \hat{s}_{c},
$$

and the fluid 4-velocity satisfies $\hat{u}_{a} \hat{s}^{a}=0$ and $\hat{s}_{a} \hat{s}^{a}=1$. Further, $\hat{\rho}$ is the energy-density function while $\hat{p}_{\perp}$ and $\hat{p}_{\|}$denote the pressure functions perpendicular and parallel to $s^{a}$, respectively. Note that for these energy-momentum tensors a perfect fluid is achieved if $p_{\|}=p_{\perp}=p$ or $\hat{p}_{\|}=\hat{p}_{\perp}=\hat{p}$ (or both). 


\subsection{General Kinematic Properties}

The kinematic properties of $\left\{M, g, u^{a}\right\}$ change in an interesting manner under the action of the minimal complex windmill transformation. Notably, the vorticity tensor is zero for $\left\{M, g, u^{a}\right\}$ while it is non-zero for $\left\{\hat{M}, \hat{g}, \hat{u}^{a}\right\}$. That is, there is a possibility of obtaining rotating purely magnetic spacetimes from irrotational purely magnetic spacetimes.

Remark 2.1. The timelike 4-velocities of $\left\{M, g, u^{a}\right\}$ and $\left\{\hat{M}, \hat{g}, \hat{u}^{a}\right\}$ are

$$
u^{a}=(0,0,0, \exp (-a))
$$

and

$$
\hat{u}^{a}=\exp (-h / 2)(0,0, \sin (k), \cos (k)),
$$

respectively.

Note that for Wainwright class B(ii) spacetimes local coordinates have been introduced such that the two Killing vectors are $\xi^{a}=(0,1,0,0)$ and $\eta^{a}=(0,0,1,0)$. Consequently the timelike 4-vector $u^{a}$ is orthogonal to these Killing vectors and invariant under the action of the symmetry group. In general, it is always possible to transform to co-moving coordinates whilst preserving the diagonal form of the metric. However, for the $G_{2} I$-B(ii) case two distinct forms of the 4-vector are possible: the canonical (co-moving) form (9) or the general (tilted) form. Since the general form is considerably more complicated the canonical form is assumed. For the windmill case this is not an issue since the only possibility is (10), see appendix A.

For $\left\{M, g, u^{a}\right\}$ the (vorticity tensor is zero, i.e. $\omega_{a c}=0$, and the) non-zero components of the shear tensor are

$$
\begin{aligned}
\sigma_{x x} & =\frac{1}{3} \exp (-a+2 b) \partial_{w}(2 b-h) \\
\sigma_{y y} & =\frac{1}{6} \exp (-a+h+2 k) \partial_{w}(-2 b+h+6 k) \\
\sigma_{z z} & =\frac{1}{6} \exp (-a+h-2 k) \partial_{w}(-2 b+h-6 k) .
\end{aligned}
$$

The non-zero component of the 4-acceleration vector $\left(\dot{u}^{a}=u^{c} \nabla_{c} u^{a}\right)$ is

$$
\dot{u}^{x}=\exp (-2 b) \partial_{x} a,
$$

and the expansion scalar is

$$
\Theta=\exp (-a) \partial_{w}(b+h) .
$$

On the other hand, for $\left\{\hat{M}, \hat{g}, \hat{u}^{a}\right\}$ the expansion scalar is 0 , i.e. $\hat{\Theta}=0$, while the non-zero components of the shear and vorticity tensors are

$$
\begin{aligned}
& \hat{\sigma}_{w u}=\frac{1}{2} \cos (\hat{k}) \exp (\hat{h} / 2) \partial_{w} \hat{k}=\hat{\omega}_{w u} \\
& \hat{\sigma}_{w v}=-\frac{1}{2} \sin (\hat{k}) \exp (\hat{h} / 2) \partial_{w} \hat{k}=\hat{\omega}_{w v} \\
& \hat{\sigma}_{x u}=\frac{1}{2} \cos (\hat{k}) \exp (\hat{h} / 2) \partial_{x} \hat{k}=\hat{\omega}_{x u} \\
& \hat{\sigma}_{x v}=-\frac{1}{2} \sin (\hat{k}) \exp (\hat{h} / 2) \partial_{x} \hat{k}=\hat{\omega}_{x v}
\end{aligned}
$$

where the vorticity scalar is

$$
\sqrt{2} \hat{\omega}=\sqrt{\exp (-2 \hat{a}(w, x))\left(\partial_{w} \hat{k}\right)^{2}+\exp (-2 \hat{b}(w, x))\left(\partial_{x} \hat{k}\right)^{2}} .
$$


Finally, the non-zero components of 4-acceleration are

$$
\begin{aligned}
& \hat{\dot{u}}^{w}=\frac{1}{2} \exp (-2 \hat{a}) \partial_{w} \hat{h} \\
& \hat{\dot{u}}^{x}=\frac{1}{2} \exp (-2 \hat{b}) \partial_{x} \hat{h} .
\end{aligned}
$$

It follows that under the action of a complex windmill transformation

$$
\{\omega=0, \Theta \neq 0\} \mapsto\{\hat{\omega} \neq 0, \hat{\Theta}=0\}
$$

while all other kinematic quantities are non-zero (in general).

Definition 2.2. A complex windmill transformation, (2), of (1) is trivial if the vorticity tensor for $\left\{\hat{M}, \hat{g}, \hat{u}^{a}\right\}$ is 0 .

\section{Non-existence results for windmill spacetimes}

In this section we show that purely magnetic windmill Einstein spaces and windmill perfect fluids in fact do not exist.

Proposition 3.1. The class of all windmill perfect fluid $(\hat{\rho}+\hat{p} \neq 0)$ spacetimes (3), with no restriction on the electric/magnetic properties of the Weyl tensor, is empty.

Proof. For the windmill spacetimes (3), assuming no restriction on the electric/magnetic properties of the Weyl tensor, suppose the energy-momentum tensor takes the form of a perfect fluid (i.e (8) with $\hat{p}_{\|}=\hat{p}_{\perp}=\hat{p} \neq-\hat{\rho}$ ) where the fluid 4-velocity is assumed to be given by (B.1). Combining

$\operatorname{ein}^{(\hat{\mathrm{pf}})}{ }_{u u}: \exp (h) \cos (k)^{2} \hat{p}+\exp (h) \sin (k)^{2} \hat{\rho}+\frac{1}{4}\left(\cos (2 k) \delta_{1}-4 \sin (2 k) \delta_{2}\right) \exp (2 a+2 b)=0$

$\operatorname{ein}^{(\hat{\mathrm{p} f})} v v: \exp (h) \sin (k)^{2} \hat{p}+\exp (h) \cos (k)^{2} \hat{\rho}-\frac{1}{4}\left(\cos (2 k) \delta_{1}-4 \sin (2 k) \delta_{2}\right) \exp (2 a+2 b)=0$, where $\delta_{1}=\left[-4 \partial_{x x} a-2 \partial_{x x} h+4 \partial_{x} a \partial_{x} b-2 \partial_{x} a \partial_{x} h+2 \partial_{x} b \partial_{x} h-4\left(\partial_{x} a\right)^{2}-\left(\partial_{x} h\right)^{2}+\right.$ $\left.4\left(\partial_{x} k\right)^{2}\right] \exp (2 a)+\left[-4 \partial_{w w} b-2 \partial_{w w} h+4 \partial_{w} a \partial_{w} b-2 \partial_{w} b \partial_{x} h+2 \partial_{w} a \partial_{w} h-4\left(\partial_{w} b\right)^{2}-\left(\partial_{w} h\right)^{2}+\right.$ $\left.4\left(\partial_{w} k\right)^{2}\right] \exp (2 b)$ and $\delta_{2}=\left[\partial_{x x} k+\partial_{x} a \partial_{x} k-\partial_{x} b \partial_{x} k+\partial_{x} h \partial_{x} k\right] \exp (2 a)+\left[\partial_{w w} k+\partial_{w} b \partial_{w} k-\right.$ $\left.\partial_{w} a \partial_{w} k+\partial_{w} h \partial_{w} k\right] \exp (2 b)$, leads to

$$
\exp (h)(\hat{\rho}+\hat{p})=0 \quad \Rightarrow \quad \hat{\rho}+\hat{p}=0,
$$

which is a contradiction. Note that the case $\hat{\rho}+\hat{p}=0$ is of no interest here as the associated class of purely magnetic spacetimes is empty, as shown below.

It should be stated that, as discussed by MacCallum [36], orthogonally transitive $G_{2} I$ spacetimes (in the form (4) of [36]) do not in general restrict the form of the energy-momentum tensor in this way and admit (3) as a particular form under given assumptions (see section 2.1).

Proposition 3.2. The class of all (non-conformally flat) Einstein spaces of $\left\{\hat{M}, \hat{g}, \hat{u}^{a}\right\}$ is empty.

Proof. For $\left\{\hat{M}, \hat{g}, \hat{u}^{a}\right\}$, suppose the Ricci tensor is of the form

$$
\hat{R}_{a c}=\hat{\Lambda} \hat{g}_{a c}
$$

where $\hat{\Lambda}$ is a constant. Also, for $\left\{\hat{M}, \hat{g}, \hat{u}^{a}\right\}$ without imposing $\hat{E}_{a c}=0$ at this stage:

$\hat{I}=-2 \exp (-4 a-2 b)\left[\left(\hat{H}_{w x}\right)^{2} \exp (2 a)+\left(\hat{H}_{w w}\right)^{2} \exp (2 b)-3\left(\hat{E}_{w w}\right)^{2} \exp (2 b)\right]$

$\hat{J}=-6 \hat{E}_{w w} \exp (-6 a-2 b)\left[\left(\hat{H}_{w x}\right)^{2} \exp (2 a)+\left(\hat{H}_{w w}\right)^{2} \exp (2 b)+\left(\hat{E}_{w w}\right)^{2} \exp (2 b)\right]$

where

$$
\begin{gathered}
\hat{H}_{w w}\left(=-\hat{H}_{x x} \exp (2 a-2 b)\right)=-\frac{1}{2}\left(-2 \partial_{w x} k+2 \partial_{w} b \partial_{x} k-\partial_{w} k \partial_{x} h-\partial_{w} h \partial_{x} k\right. \\
\left.+2 \partial_{x} a \partial_{w} k\right) \exp (a-b)
\end{gathered}
$$




$$
\begin{gathered}
\hat{H}_{w x}=\frac{1}{2}\left(\partial_{w w} k-\partial_{w} b \partial_{w} k+\partial_{w} h \partial_{w} k-\partial_{w} a \partial_{w} k\right) \exp (-a+b) \\
-\frac{1}{2}\left(\partial_{x x} k-\partial_{x} a \partial_{x} k+\partial_{x} h \partial_{x} k-\partial_{x} b \partial_{x} k\right) .
\end{gathered}
$$

Note that for $\left\{M, g, u^{a}\right\}$, the non-zero component of $H_{a c}$ is

$$
H_{y z}=\exp (-2 a+h) \hat{H}_{w w} \text {. }
$$

Now, (recall $\hat{E}_{a c}=0$ ) by $\hat{E}_{w w}=0$ (or equivalently by any other constraint; $\hat{E}_{x x}=0$ or $\hat{E}_{u u}=0$ or $\hat{E}_{u v}=0$ or $\hat{E}_{v v}=0$ ),

$$
\begin{aligned}
\partial_{w w} h-\partial_{w} a \partial_{w} h & +\partial_{w} b \partial_{w} h+2 \partial_{w} a \partial_{w} b-2 \partial_{w w} b-4\left(\partial_{w} k\right)^{2}-2\left(\partial_{w} b\right)^{2} \\
& +\left[\partial_{x x} h-\partial_{x} b \partial_{x} h+\partial_{x} a \partial_{x} h+2 \partial_{x} b \partial_{x} a-2 \partial_{x x} a-4\left(\partial_{x} k\right)^{2}-2\left(\partial_{x} a\right)^{2}\right] \\
& \times \exp (2 a-2 b)=0 .
\end{aligned}
$$

Note that this expression will also be required in the next section.

It follows that, with constraint (29) identically satisfied, the Weyl invariant [22] $\hat{J}=0$ (and $\hat{I} \in \mathbb{R}$ ) and therefore the spacetime is of Petrov-type $I\left(M^{\infty}\right)$. A theorem due to Brans [39] (also see Szekeres [40]), which can be trivially extended to Einstein spaces (see Barnes [41]), then implies that this spacetime is conformally-flat, a contradiction to assumption 2.1.3. Note that the core of this proof is (25), which more generally shows that any purely magnetic windmill spacetime is of type $I\left(M^{\infty}\right)$ in the extended Weyl-Petrov type classification by Arianrhod and McIntosh [42].

\section{4. $G_{2} I$-B(ii) PMpf $\leftrightarrow$ windmill PMaf}

In this section, as a result of the analysis of the previous section, a minimal complex windmill transformation of $G_{2} I$-B(ii) PMpf spacetimes is considered.

\section{1. $G_{2} I-B(i i) P M p f$}

For $\left\{M, g, u^{a}\right\}$ the constraints $E_{x x}=0$ and $E_{y y}=0$ (which together satisfy $E_{z z}=0$ ) are $\left[-\partial_{w} b \partial_{w} h+2 \partial_{w} b^{2}-2 \partial_{w} b \partial_{w} a-4\left(\partial_{w} k\right)^{2}-\partial_{w w} h+2 \partial_{w w} b+\partial_{w} a \partial_{w} h\right] \exp (-2 a+2 b)$

$$
+\partial_{x x} h-\partial_{x} b \partial_{x} h+4\left(\partial_{x} k\right)^{2}+2 \partial_{x} b \partial_{x} a-2\left(\partial_{x} a\right)^{2}-2 \partial_{x x} a+\partial_{x} a \partial_{x} h=0
$$

and

$$
\begin{aligned}
{\left[-\partial_{w} b \partial_{w} k-\right.} & \frac{1}{3}\left(\partial_{w} b\right)^{2}-\frac{1}{3} \partial_{w w} b+\frac{1}{3} \partial_{w} b \partial_{w} a+\frac{2}{3}\left(\partial_{w} k\right)^{2}+\frac{1}{6} \partial_{w w} h \\
& \left.+\partial_{w w} k-\partial_{w} a \partial_{w} k+\frac{1}{6} \partial_{w} b \partial_{w} h-\frac{1}{6} \partial_{w} a \partial_{w} h+\partial_{w} h \partial_{w} k\right] \exp (-2 a+2 b) \\
& +\partial_{x x} k+\frac{1}{3}\left(\partial_{x} a\right)^{2}+\frac{1}{6} \partial_{x} b \partial_{x} h-\frac{1}{6} \partial_{x x} h-\frac{2}{3}\left(\partial_{x} k\right)^{2}-\partial_{x} b \partial_{x} k \\
& -\partial_{x} a \partial_{x} k+\frac{1}{3} \partial_{x x} a+\partial_{x} h \partial_{x} k-\frac{1}{3} \partial_{x} b \partial_{x} a-\frac{1}{6} \partial_{x} a \partial_{x} h=0
\end{aligned}
$$

respectively.

Furthermore, a perfect fluid is established if the following field equations are satisfied:

$$
\begin{aligned}
&\left(\operatorname{ein}_{y y}^{(\mathrm{pf})}\right) \quad \partial_{x x} h+2 \partial_{x} b \partial_{x} k-\partial_{x} a \partial_{x} h-2 \partial_{x} b \partial_{x} a+2\left(\partial_{x} a\right)^{2}+4\left(\partial_{x} k\right)^{2}-\partial_{x} b \partial_{x} h \\
&-2 \partial_{x x} k-2 \partial_{x} a \partial_{x} k+2 \partial_{x x} a-2 \partial_{x} h \partial_{x} k \\
&+\left[2 \partial_{w} h \partial_{w} k+2 \partial_{w} b \partial_{w} k-\partial_{w} a \partial_{w} h+2 \partial_{w} b \partial_{w} a-2\left(\partial_{w} b\right)^{2}-2 \partial_{w w} b\right. \\
&\left.-\partial_{w} b \partial_{w} h+\partial_{w w} h+2 \partial_{w w} k-2 \partial_{w} a \partial_{w} k+\left(\partial_{w} h\right)^{2}\right] \exp (-2 a+2 b)=0, \\
&\left(\operatorname{ein}^{(\mathrm{pf})} z z\right) \quad \partial_{w w} h-2 \partial_{w} h \partial_{w} k-2\left(\partial_{w} b\right)^{2}-2 \partial_{w w} b-\partial_{w} b \partial_{w} h-\partial_{w} a \partial_{w} h \\
&+2 \partial_{w} b \partial_{w} a+2 \partial_{w} a \partial_{w} k+\left(\partial_{w} h\right)^{2}-2 \partial_{w w} k-2 \partial_{w} b \partial_{w} k \\
&+\left[-2 \partial_{x} b \partial_{x} k+2 \partial_{x} a \partial_{x} k+4\left(\partial_{x} k\right)^{2}-\partial_{x} b \partial_{x} h-\partial_{x} a \partial_{x} h+2 \partial_{x} h \partial_{x} k\right. \\
&\left.+2\left(\partial_{x} a\right)^{2}+2 \partial_{x x} a+2 \partial_{x x} k-2 \partial_{x} b \partial_{x} a+\partial_{x x} h\right] \exp (2 a-2 b)=0
\end{aligned}
$$


and

$$
\left(\operatorname{ein}_{w x}^{(\mathrm{pf})}\right) \quad \partial_{w x} h-\partial_{w} b \partial_{x} h+\frac{1}{2} \partial_{x} h \partial_{w} h+2 \partial_{x} k \partial_{w} k-\partial_{x} a \partial_{w} h=0 .
$$

In addition, the energy-density and pressure functions are

$$
\begin{aligned}
\rho=\left[\partial_{x} b \partial_{x} h-\right. & \left.\partial_{x x} h-\frac{3}{4}\left(\partial_{x} h\right)^{2}-\left(\partial_{x} k\right)^{2}\right] \exp (-2 b) \\
+ & {\left[\frac{1}{4}\left(\partial_{w} h\right)^{2}+\partial_{w} b \partial_{w} h-\left(\partial_{w} k\right)^{2}\right] \exp (-2 a) } \\
p=\left[\partial_{x} a \partial_{x} h+\right. & \left.\frac{1}{4}\left(\partial_{x} h\right)^{2}-\left(\partial_{x} k\right)^{2}\right] \exp (-2 b) \\
+ & {\left[-\frac{3}{4}\left(\partial_{w} h\right)^{2}+\partial_{w} a \partial_{w} h-\partial_{w w} h-\left(\partial_{w} k\right)^{2}\right] \exp (-2 a) }
\end{aligned}
$$

subject to the conservation equations. Finally, recall that the only non-zero component of $H_{a c}$ is (28).

If either the acceleration vector or the spatial derivative of the energy-density function is 0 then the WVL spacetime is recovered. Recall, the fluid 4-velocity (9) is irrotational (see section 2.3), the non-zero component of the 4-acceleration vector is (14) and the spatial derivative $\left(D_{a} A_{b_{1} \ldots b_{n}} \equiv h_{a}^{c} h_{b_{1}}^{d_{1}} \ldots h_{b_{n}}^{d_{n}} \nabla_{c} A_{d_{1} \ldots d_{n}}\right.$ where $h_{a b}=g_{a b}+u_{a} u_{b}$, see Maartens [43]) of the energy-density function is

$$
D_{x} \rho=6 H_{y z} \exp (-h) \partial_{w} k .
$$

Note that it is not known if the class of irrotational, Petrov-type $I$, PMpf spacetimes where $D_{a} \rho \neq 0$ and $\dot{u}^{a} \neq 0$ is empty or non-empty.

Partial decoupling of equations. Before proceeding it is beneficial to manipulate (29), (30), (31), (32) and (33) in the following way: combining (30), (31) and (32) produces:

$$
\partial_{w w} k=\partial_{w} a \partial_{w} k+\exp (2 a-2 b) \partial_{x} a \partial_{x} k-\partial_{w} h \partial_{w} k
$$

and then, in turn, (38) leads to

$\partial_{x x} k=\partial_{x} b \partial_{x} k+\exp (2 b-2 a) \partial_{w} b \partial_{w} k-\partial_{x} h \partial_{x} k$

$\partial_{x x} h=\partial_{x} b \partial_{x} h-4\left(\partial_{x} k\right)^{2}+\left[2\left(\partial_{w} k\right)^{2}+\partial_{w} b \partial_{w} h-\frac{1}{2}\left(\partial_{w} h\right)^{2}\right] \exp (-2 a+2 b)$.

Now, combining (29) and (33) given (38)-(40), leads to

$$
\begin{gathered}
\partial_{w w} h=2\left(\partial_{w} b\right)^{2}+2 \partial_{w w} b+\partial_{w} a \partial_{w} h-2 \partial_{w} b \partial_{w} a+4 \exp (-2 b+2 a)\left(\partial_{x} k\right)^{2}-\partial_{w} b \partial_{w} h \\
\partial_{x x} a=\partial_{x} b \partial_{x} a-2\left(\partial_{x} k\right)^{2}-\left(\partial_{x} a\right)^{2}+\frac{1}{2} \partial_{x} a \partial_{x} h \\
+\left[-\left(\partial_{w} k\right)^{2}-\frac{1}{4}\left(\partial_{w} h\right)^{2}+\frac{1}{2} \partial_{w} b \partial_{w} h\right] \exp (-2 a+2 b) .
\end{gathered}
$$

\subsection{Windmill PMaf}

Recall, for $\left\{\hat{M}, \hat{g}, \hat{u}^{a}\right\}, \hat{E}_{a c}=0$ is satisfied by (29) and the non-zero components of $\hat{H}_{a c}$ are given by (26) and (27).

Now, consider the form of the energy-momentum tensor. For a general unit spacelike 4-vector

$$
\begin{aligned}
& \hat{\mathfrak{S}}^{a}=\frac{1}{\sqrt{\mathcal{S}}}\left(\hat{\mathfrak{s}}^{w}, \hat{\mathfrak{s}}^{x}, \hat{\mathfrak{s}}^{u},-\tan (k) \hat{\mathfrak{s}}^{u}\right), \\
& \left(\operatorname{ein}^{(\widehat{\mathrm{af}})}{ }_{w u} \text { or } \quad \operatorname{ein}^{(\widehat{\mathrm{af}})}{ }_{w v}\right) \quad \hat{\mathfrak{s}}^{w} \hat{\mathfrak{s}}^{u}\left(\hat{p}_{\perp}-\hat{p}_{\|}\right)=0,
\end{aligned}
$$




$$
\left(\operatorname{ein}^{(\widehat{a f f})}{ }_{x u} \text { or } \quad \operatorname{ein}^{\left(\widehat{a ̂ f}{ }_{x v}\right.}\right) \quad \hat{\mathfrak{s}}^{x} \hat{\mathfrak{s}}^{u}\left(\hat{p}_{\perp}-\hat{p}_{\|}\right)=0
$$

and therefore (by proposition $3.1 \hat{p}_{\perp} \neq \hat{p}_{\|}$)

$$
\hat{\mathfrak{S}}^{a}= \begin{cases}\frac{1}{\sqrt{\mathcal{S}}}\left(\hat{\mathfrak{s}}^{w}, \hat{\mathfrak{s}}^{x}, 0,0\right) & \text { If } \hat{\mathfrak{s}}^{u}=0 \\ \frac{\hat{\mathfrak{s}}^{u}}{\sqrt{\mathcal{S}}}(0,0,1,-\tan (k)) & \text { If } \hat{\mathfrak{s}}^{w}=0=\hat{\mathfrak{s}}^{x},\end{cases}
$$

where $\mathcal{S}$ is the normalization for $\hat{s}^{a}$.

4.2.1. $\hat{\mathfrak{s}}^{u}=0 . \quad$ In this case, an anisotropic fluid is established if

$$
\begin{array}{cc}
\left(\operatorname{ein}^{(\widehat{\widehat{a f}})}{ }_{w x}\right) & 16 \partial_{x} k \partial_{w} k+\frac{[\alpha-\beta] \hat{\mathfrak{s}}^{x} \hat{\mathfrak{s}}^{w}}{\left[\exp (b) \hat{\mathfrak{s}}^{x}-\hat{\mathfrak{s}}^{w} \exp (a)\right]\left[\exp (b) \hat{\mathfrak{s}}^{x}+\hat{\mathfrak{s}}^{w} \exp (a)\right]}=0 \\
\left(\operatorname{ein}{ }^{(\widehat{\mathrm{af}})}{ }_{u v}\right) \quad 8\left[\exp (2 a) \partial_{x} a \partial_{x} k+\exp (2 b) \partial_{w} b \partial_{w} k\right] \\
-\frac{\left[\left(\hat{\mathfrak{s}}^{x}\right)^{2} \alpha \exp (2 b)-\left(\hat{\mathfrak{s}}^{w}\right)^{2} \beta \exp (2 a)\right] \sin (k)}{\left[\left(\hat{\mathfrak{s}}^{w}\right)^{2} \exp (2 a)-\exp (2 b)\left(\hat{\mathfrak{s}}^{x}\right)^{2}\right] \cos (k)}=0
\end{array}
$$

$\left(\right.$ ein $\left.{ }^{(\widehat{\mathrm{af}})}{ }_{u u}\right) \quad 8\left[\exp (2 a) \partial_{x} a \partial_{x} k+\exp (2 b) \partial_{w} b \partial_{w} k\right]$

$$
+\frac{\left[\left(\hat{\mathfrak{s}}^{x}\right)^{2} \alpha \exp (2 b)-\left(\hat{\mathfrak{s}}^{w}\right)^{2} \beta \exp (2 a)\right] \cos (2 k)}{\left[\left(\hat{\mathfrak{s}}^{w}\right)^{2} \exp (2 a)-\exp (2 b)\left(\hat{\mathfrak{s}}^{x}\right)^{2}\right] \sin (2 k)}=0
$$

where $\left(\hat{\mathfrak{s}}^{w}\right)^{2} \exp (2 a)-\exp (2 b)\left(\hat{\mathfrak{s}}^{x}\right)^{2} \neq 0$ and

$$
\begin{aligned}
& \alpha=2\left[-4\left(\partial_{x} k\right)^{2}-2 \partial_{x} a \partial_{x} h+\left(\partial_{x} h\right)^{2}\right] \exp (2 a) \\
& +4\left[-2 \partial_{w w} b+2 \partial_{w} a \partial_{w} b+\partial_{w} b \partial_{w} h-2\left(\partial_{w} b\right)^{2}+4\left(\partial_{w} k\right)^{2}\right] \exp (2 b) \\
& \beta=4 \exp (2 b) \partial_{w} h \partial_{w}(-2 b+h)+32 \exp (2 a)\left(\partial_{x} k\right)^{2} .
\end{aligned}
$$

However, combining (47) and (48) yields

$$
\exp (2 a) \partial_{x} a \partial_{x} k+\exp (2 b) \partial_{w} b \partial_{w} k=0
$$

and

$$
\left(\hat{\mathfrak{s}}^{x}\right)^{2} \alpha \exp (2 b)-\left(\hat{\mathfrak{s}}^{w}\right)^{2} \beta \exp (2 a)=0 .
$$

That is, (51) and (52) can now replace (47) and (48) entirely.

Lemma 4.1. For all Windmill PMaf spacetimes assuming the special cases $\hat{\mathfrak{s}}^{w}=0$ or $\hat{\mathfrak{s}}^{x}=0$ leads to separable solutions.

Proof. Suppose that either $\hat{\mathfrak{s}}^{x}=0$ or $\hat{\mathfrak{s}}^{w}=0$ then, by (46),

$$
\partial_{w} k \partial_{x} k=0 .
$$

The case $\partial_{w} k=0 \Rightarrow D_{x} \rho=0$ and leads to the WVL PMpf that will be considered in the next section. Here, it is assumed that (53) is satisfied by $\partial_{x} k=0\left(\neq \partial_{w} k\right)$. Note, for the same reason, it is also assumed that $\partial_{x} a \neq 0$ (see (14)). In turn, by (51), $\partial_{w} k \partial_{w} b=0 \Rightarrow \partial_{w} b=0$. These conditions reduce (34), (38) and (41) to the form

$$
\begin{aligned}
& \partial_{x}\left(\partial_{w} h\right)=\partial_{x}\left(a-\frac{1}{2} h\right) \partial_{w} h, \\
& \partial_{w}\left(\partial_{w} k\right)=\partial_{w}(a-h) \partial_{w} k,
\end{aligned}
$$


and

$$
\partial_{w}\left(\partial_{w} h\right)=\partial_{w} a \partial_{w} h
$$

respectively.

The general solution of (54)-(56) could be obtained in a straightforward manner. Substituting the first derivative with respect to $w$ of the general solution of (56),

$$
\partial_{w} h=v_{1} \exp (a), \quad\left[v_{1}=v_{1}(x)\right]
$$

into (54) reduces this equation to the form $\partial_{x}\left(h+2 v_{1}\right)=0 \Rightarrow h=-2 v_{1}+\mu_{1}\left[\mu_{1}=\mu_{1}(w)\right]$. Then comparing this result with (57) leads to

$$
a=-v_{1}+\ln \partial_{w} \mu_{1}
$$

where $\partial_{w} \mu_{1} \neq 0$. By a suitable coordinate transformation of the $G_{2} I$ metric, such that $w \equiv \mu_{1}$, these functions can be simplified to the form

$$
\begin{aligned}
& a=-v_{1} \\
& h=-2 v_{1}+w .
\end{aligned}
$$

Now, since $a-h=v_{1}-w^{7},(55)$ reduces to

$$
d_{w}\left(d_{w} k\right)=-d_{w} k
$$

with general solution

$$
k=-\exp \left(c_{1}-w\right)
$$

(recall $\partial_{x} k=0$ ). Note that an integration constant was absorbed by suitable coordinate transformation. However, combining (40) and (42), given (59), (60) and (62), leads to

$$
0=-d_{x} b d_{x} v_{1}+2\left(d_{x} v_{1}\right)^{2}+4 \exp \left\{2\left(c_{1}-w\right)\right\} \exp \left\{2\left(b+v_{1}\right)\right\}
$$

which cannot be satisfied for any $b$ (recall $\left.\partial_{w} b=0\right)$ and $v_{1}$.

On the other hand, suppose (54) and (56) are satisfied by $\partial_{w} h=0 \Rightarrow h=v_{3}$ then (55) reduces to

$$
d_{w}\left(d_{w} k\right)=d_{w} a d_{w} k
$$

with general solution $\exp (a)=\exp \left(v_{4}\right) d_{w} k$. However, by a suitable coordinate transformation, $a=v_{4}$ and, as a result, $k=w$. The next step is to choose coordinates such that $a=b=v_{4}$ then (40) and (42) reduce to a pair of coupled ODEs

$$
\begin{aligned}
& d_{x x} v_{3}=d_{x} v_{4} d_{x} v_{3}+2 \\
& d_{x x} v_{4}=\frac{1}{2} d_{x} v_{4} d_{x} v_{3}-1
\end{aligned}
$$

Note that, by (49), (50) and (52), $\beta=0$ and therefore $\hat{\mathfrak{s}}^{x}=0$ and $\alpha \neq 0$ otherwise both the $G_{2} I$-B(ii) (1) and windmill (3) spacetimes are conformally flat, a contradiction.

Solutions of (65) and (66) determine the spacetime by effectively defining the conformal factor, $\exp (2 \Omega)=\exp \left(2 \int \Upsilon \mathrm{d} x\right)$ where $\Upsilon \equiv d_{x} v_{4}$. That is, combining (65) and (66) produces

$$
\begin{aligned}
& d_{x} \nu_{3}-2 \Upsilon=4 x+c_{2}, \\
& d_{x} \Upsilon-\Upsilon^{2}-\left(2 x+c_{3}\right) \Upsilon=-1
\end{aligned}
$$

where $c_{3}=c_{2} / 2$. The Riccati equation (68) can be transformed, by

$$
\Xi=\exp \left(-\int \Upsilon \mathrm{d} x\right),
$$

7 Note that by $\partial_{x} k=0$ it immediately follows from (55) that $a-h$ is a separable function of the coordinates $w$ and $x$. 
to a second-order linear ODE:

$$
d_{x x} \Xi-\left(2 x+c_{3}\right) d_{x} \Xi-\Xi=0 .
$$

The general solution of (70) is expressed in terms of the confluent hypergeometric (Kummer's) functions $M\left(\equiv_{1} F_{1}\right)=M\left(n_{1}, n_{2} ; x\right)$ and $U=U\left(n_{1}, n_{2} ; x\right)$ :

$$
\Xi=c_{5} M\left(\frac{1}{4}, \frac{1}{2},\left(x+c_{3} / 2\right)^{2}\right)+c_{6} U\left(\frac{1}{4}, \frac{1}{2},\left(x+c_{3} / 2\right)^{2}\right) .
$$

It follows from (69) that

$$
\Upsilon=-d_{x} \ln (\Xi)
$$

Therefore, from the analysis above the solution set for the spacetime is given by

$$
\{a, b, h, k\}=\left\{-\ln (\Xi),-\ln (\Xi),-2 \ln (\Xi)+2 x^{2}+2 c_{3} x, w\right\}
$$

and the energy-density functions and pressure functions are

$$
\begin{aligned}
& \rho=-3\left(4 x^{2}+4 c_{3} x+c_{3}^{2}+1\right) \Xi^{2}-3\left(d_{x} \Xi\right)^{2}+6\left(2 x+c_{3}\right) \Xi d_{x} \Xi \\
& p=\left(2 x+c_{3}+1\right)\left(2 x+c_{3}-1\right) \Xi^{2}+3\left(d_{x} \Xi\right)^{2}-4\left(2 x+c_{3}\right) \Xi d_{x} \Xi \\
& \hat{\rho}=-\hat{p}_{\perp}=-p \\
& \hat{p}_{\|}=-\rho .
\end{aligned}
$$

Note that $\hat{s}^{a}=(\Xi, 0,0,0)$ and by (74)-(77)

$$
\hat{T}_{a c}^{(\mathrm{af})}=-p \hat{g}_{a c}-(\rho+p) \hat{s}_{a} \hat{s}_{c}
$$

and the vorticity scalar $\hat{\omega}=\Xi / \sqrt{2} \neq 0$.

Proposition 4.1. For all Windmill PMaf spacetimes the assumption of separation of variables leads to the special cases $\hat{\mathfrak{s}}^{w}=0$ or $\hat{\mathfrak{s}}^{x}=0$.

Proof. As seen above, the special case $\hat{\mathfrak{s}}^{x}=0$ or $\hat{\mathfrak{s}}^{w}=0$ led naturally to solutions separable in the variables $\{a, b, h, k\}$. Note that the WVL PMpf is separable in co-moving coordinates. Here, separation of these variables is considered from the outset and it is assumed that $\partial_{w} k \partial_{x} k \neq 0$.

Let

$$
\begin{aligned}
& a=a_{0}(w)+a_{1}(x) \\
& b=b_{0}(w)+b_{1}(x) \\
& h=h_{0}(w)+h_{1}(x) \\
& k=k_{0}(w)+k_{1}(x)
\end{aligned}
$$

and then choose coordinates such that $a=b$ or $f_{0} \equiv a_{0}=b_{0}$ and $f_{1} \equiv a_{1}=b_{1}$. This reduces (41), and a combination of (40) and (42), to the form

$$
\begin{aligned}
& d_{w w}\left(2 f_{0}-h_{0}\right)+4\left(d_{x} k_{1}\right)^{2}=0 \\
& d_{x x}\left(2 f_{1}-h_{1}\right)+4\left(d_{w} k_{0}\right)^{2}=0
\end{aligned}
$$

respectively. These equations imply

$$
\begin{aligned}
& k_{0}=c_{10} w \\
& k_{1}=c_{11} x
\end{aligned}
$$




$$
\begin{aligned}
& 2 f_{0}-h_{0}=-4 c_{10}^{2} w^{2}+c_{12} w \\
& 2 f_{1}-h_{1}=-4 c_{11}^{2} x^{2}+c_{13} x
\end{aligned}
$$

where all integration constants have been absorbed by suitable coordinate transformations.

Finally, consider (51) subject to (79):

$$
\left(d_{w} f_{0}\right)\left(d_{w} k_{0}\right)+\left(d_{x} f_{1}\right)\left(d_{x} k_{1}\right)=0 .
$$

Substituting (82)-(85) into (86) leads to

$$
\begin{aligned}
& h_{0}=8 c_{10}^{2} w^{2}+c_{14} w \\
& h_{1}=8 c_{11}^{2} x^{2}+c_{15} x
\end{aligned}
$$

where $c_{11}\left(c_{12}+c_{14}\right)+c_{10}\left(c_{13}+c_{15}\right)=0$. However, by (39) (or similarly (38)) subject to (82)-(85) and (87)-(88):

$$
-8 c_{10} c_{11}^{2} x+\frac{1}{2}\left[c_{10}\left(c_{13}+c_{15}\right)+c_{11}\left(c_{12}+c_{14}\right)-2 c_{10} c_{15}\right]=0
$$

which implies $c_{10} c_{11}=0 \Rightarrow \partial_{w} k \partial_{x} k=0$ and therefore assuming separation of variables yields the solutions found above.

4.2.2. $\hat{\mathfrak{s}}^{w}=0=\hat{\mathfrak{s}}^{x}$ (and $\hat{\mathfrak{s}}^{u} \neq 0$ ). In this case, an anisotropic fluid is established if

$$
\begin{array}{ll}
\left(\operatorname{ein}^{(\widehat{\mathrm{af}})}{ }_{w x}\right) & \partial_{x} k \partial_{w} k=0, \\
\left(\operatorname{ein}^{(\widehat{\mathrm{af}})}\right) & \exp (2 a) \partial_{x} a \partial_{x} k+\exp (2 b) \partial_{w} b \partial_{w} k=0, \\
\left(\operatorname{ein}^{(\widehat{\mathrm{af}})}{ }_{x x}\right) \quad & 2 \partial_{x x} h+\left(\partial_{x} h\right)^{2}-4\left(\partial_{x} k\right)^{2}-2 \partial_{x} b \partial_{x} h-2 \partial_{x} a \partial_{x} h \\
& -\left[2 \partial_{w w} h+\left(\partial_{w} h\right)^{2}-4\left(\partial_{w} k\right)^{2}-2 \partial_{w} a \partial_{w} h-2 \partial_{w} b \partial_{w} h\right] \exp (-2 a+2 b)=0 .
\end{array}
$$

Recall, from above, the case $\partial_{w} k=0$ or $\partial_{x} a=0$ could lead to the WVL PMpf (see section 5). Therefore, it is assumed that (90) and, in turn, (91) are satisfied by $\partial_{x} k=0=\partial_{w} b$ (with $\partial_{w} k \neq 0 \neq \partial_{x} a$ ). It follows that (55)-(57) also holds in this case, and by the same argument as for the case $\hat{\mathfrak{s}}^{u}=0$ it follows that $\partial_{w} h=0$. However, by comparing (40) with (92) and taking $x$-derivatives, one can easily show that this case is inconsistent. Hence, there are no solutions with $\hat{\mathfrak{s}}^{w}=\hat{\mathfrak{s}}^{x}=0$.

\section{PMaf generalizations of the WVL spacetime}

As stated in the previous section, if $\partial_{w} k=0 \Rightarrow a_{x}=0$, then, by a theorem due to Wylleman and Van den Bergh [34], a unique PMpf is obtained.

The solution set for the WVL PMpf is

$\{a, b, h, k\}=\left\{-w^{2}-\ln (w)-\ln (C / 2),-w^{2}-\ln (w)-\ln (C / 2),-2 \ln (w)-2 \ln (C / 2), x\right\}$

where

$$
\begin{aligned}
& u^{a}=\frac{1}{2} C w \exp \left(w^{2}\right)(0,0,0,1) \\
& \rho=\frac{3}{4} C^{2}\left(w^{2}+1\right) \exp \left(2 w^{2}\right)
\end{aligned}
$$




$$
p=\frac{3}{4} C^{2}\left(w^{2}-1\right) \exp \left(2 w^{2}\right) .
$$

For the related PMaf

$$
\begin{aligned}
& \hat{u}^{a}=\frac{1}{2} C w(0,0, \sin (x), \cos (x)), \\
& \hat{s}^{a}=\frac{1}{2} C w \exp \left(w^{2}\right)(1,0,0,0), \\
& \hat{\rho}=-\hat{p}_{\perp}=p, \\
& \hat{p}_{\|}=\rho
\end{aligned}
$$

and the vorticity scalar $\hat{\omega}=C w \exp \left(w^{2}\right) /(2 \sqrt{2}) \neq 0$.

At first glance, it would appear that the solution set for the WVL PMpf spacetime may, in some way, be related to the PMpf derived in section 4.2.1 (Cf (73)) — by simply interchanging the $w$ and $x$ coordinates (coordinate interchange symmetry [32]) up to the introduction of an appropriate conformal factor. To attempt this, first consider the spacetime for (73):

$g=\exp \left(2 \Omega^{\prime}\right)\left[+\mathrm{d} x^{2}+\exp \left(2 x^{2}+\gamma x\right)\left\{\exp (2 w) \mathrm{d} y^{2}+\exp (-2 w) \mathrm{d} z^{2}\right\}-\mathrm{d} w^{2}\right]$

where $\gamma \equiv c_{3}$ and the conformal factor is redefined to be an arbitrary function of $w$ and $x$ only. It follows that, for the metric coefficients of (101), interchanging $w \leftrightarrow x$ produces

$g=\exp \left(2 \Omega^{\prime}\right)\left[+\mathrm{d} x^{2}+\exp \left(2 w^{2}+\gamma w\right)\left\{\exp (2 x) \mathrm{d} y^{2}+\exp (-2 x) \mathrm{d} z^{2}\right\}-\mathrm{d} w^{2}\right]$.

The conformal function is then defined to be

$$
\Omega^{\prime}=-w^{2}-\frac{1}{2} \gamma w-\ln (w)-\ln \left(C^{\prime}\right)
$$

and this leads to

$C^{\prime 2} g=w^{-2} \exp \left(-2 w^{2}-\gamma w\right)\left[-\mathrm{d} w^{2}+\mathrm{d} x^{2}\right]+w^{-2}\left[\exp (2 x) \mathrm{d} y^{2}+\exp (-2 x) \mathrm{d} z^{2}\right]$

where $C^{\prime}=C / 2$ and $C$ is the WVL constant scaling parameter.

Remarkably, this is a one parameter PMaf generalization of the WVL PMpf spacetime which is recovered by setting $\gamma=0$. The fluid 4-velocity is given by (9) with $a=\Omega^{\prime}$, $s^{a}=(C w \exp \{w(2 w+\gamma)\}, 0,0,0)$ :

$$
\begin{aligned}
& \rho=\frac{1}{4} C^{2}\left(3 w^{2}+\gamma w+3\right) \exp \{w(2 w+\gamma)\}, \\
& p_{\perp}=\frac{3}{4} C^{2}\left(w^{2}-1\right) \exp \{w(2 w+\gamma)\}, \\
& p_{\|}=\frac{1}{4} C^{2}\left(3 w^{2}+\gamma w-3\right) \exp \{w(2 w+\gamma)\} .
\end{aligned}
$$

In addition, (104) is related, by a minimal complex windmill transformation, to a purely magnetic windmill spacetime albeit with an energy-momentum tensor beyond the scope of this study.

\section{Discussion and conclusion}

It turns out that real slices of a complexified $G_{2} I$-B(ii) spacetime, subject to assumptions 2.1, lead to two distinct PMpf spacetimes, i.e. the WVL PMpf (93) and the new solution (73), both with related windmill PMaf spacetimes. It follows that this approach can be viewed in two important ways: as a generating method, leading to a new/known spacetime from a known 'seed' spacetime or a method that may produce (and thereby relate) two completely new spacetimes. 


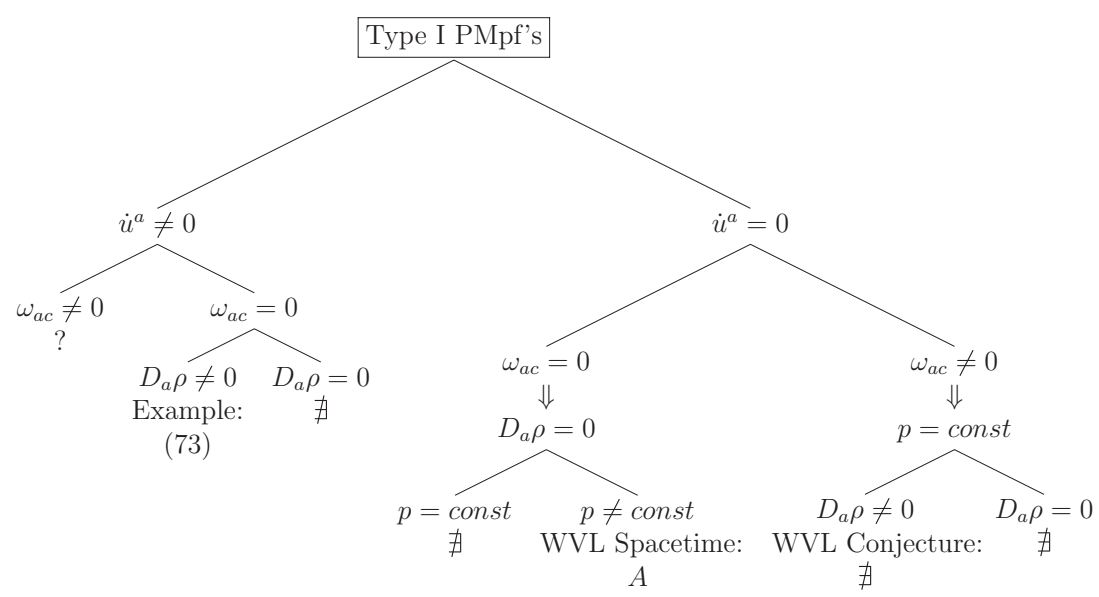

Figure 1. Classification tree for Petrov type I PMpfs. Here $\nexists$ indicates inconsistency, while $A$ means that all solutions are known.

Among the assumptions made in this study, it was assumed that the energy-momentum tensor for each real spacetime was in the form of an anisotropic or perfect fluid while the Weyl tensor was of the purely magnetic type. However, there is no reason why further investigations could not consider other interesting possibilities; in particular, vacuum purely electric spacetimes. Furthermore, the assumptions on the Ricci or Weyl tensors could be removed entirely. That is, for example, only vacuum spacetimes could be considered, although a complete integration of the resulting equations may be formidable.

Figure 1 shows the classification tree for Petrov type I PMpf's, which is a refinement of the one presented in [34].

\section{Acknowledgments}

CL thanks Michael Bradley, Kjell Rosquist and Norbert Van den Bergh and for many interesting discussions. LW is supported by a BOF research grant (UGent). We also thank the anonymous referees for helpful comments.

\section{Appendix A. windmill spacetime signature}

The windmill spacetime can be written as

$$
\hat{g}=\epsilon_{1} \exp (2 \hat{a}) \mathrm{d} w^{2}+\epsilon_{2} \exp (2 \hat{b})\left(\mathrm{d} x^{2}\right)+\epsilon_{3} \exp (\hat{h}) \hat{g}^{(2)}
$$

where $\hat{g}^{(2)}=\cos (2 \hat{k})\left(\mathrm{d} u^{2}-\mathrm{d} v^{2}\right)-2 \sin (2 \hat{k}) \mathrm{d} u \mathrm{~d} v$.

It follows that the timelike $(\mathcal{T})$-spacelike $(\mathcal{S})$ nature of the coordinates $u$ and $v$ are defined on the principal branch:

$\mathcal{P}_{v} \cup \mathcal{P}_{u}=\{\hat{k} \in(0, \pi / 4) \mid v \in \mathcal{T}$ and $u \in \mathcal{S}\} \cup\{\hat{k} \in(\pi / 4, \pi / 2) \mid v \in \mathcal{S}$ and $u \in \mathcal{T}\}$.

Note that the determinant of $\hat{g}^{(2)}$ is

$$
\left|\hat{g}^{(2)}\right|=\left|\begin{array}{cc}
\cos (2 \hat{k}) & -\sin (2 \hat{k}) \\
-\sin (2 \hat{k}) & -\cos (2 \hat{k})
\end{array}\right|=-1 .
$$

Note also that, the spacetime signature can always be preserved by the set of constants $\epsilon_{1}, \epsilon_{2}, \epsilon_{3} \in\{-1,+1\}$. Note that for the 4-velocity (10) on $\mathcal{P}_{v} \cup \mathcal{P}_{u} \epsilon_{3}=+1$. 


\section{Appendix B. Unit timelike 4-vector $\hat{\boldsymbol{u}}^{a}$}

On the other hand, for $\left\{\hat{M}, \hat{g}, \hat{u}^{a}\right\}$, the electric and magnetic parts of the Weyl tensor, with respect to

$$
\hat{u}^{a}=\frac{1}{\sqrt{\hat{\mathcal{U}}}}\left(0,0, \hat{\mathfrak{u}}^{u}, \hat{\mathfrak{u}}^{v}\right)
$$

where

$$
\hat{\mathcal{U}}=\left\{\cos (2 k)\left[\left(\hat{\mathfrak{u}}^{v}\right)^{2}-\left(\hat{\mathfrak{u}}^{u}\right)^{2}\right]+2 \sin (2 k) \hat{\mathfrak{u}}^{u} \hat{\mathfrak{u}}^{v}\right\} \exp (h / 2),
$$

are

$$
\begin{aligned}
& \hat{E}_{w w}=\frac{\beta_{3} \beta_{4} \exp (h)}{3 \mathcal{U} \cos (2 k)} \\
& \hat{E}_{w x}=-\frac{3 \beta_{2}}{\beta_{3}} \cos (2 k) \hat{E}_{w w} \\
& \hat{E}_{x x}=-\hat{E}_{w w}
\end{aligned}
$$

and

$$
\begin{aligned}
& \hat{H}_{w w}=\frac{1}{2 \mathcal{U}} \beta_{2}\left(\left(\mathfrak{u}^{u}\right)^{2}+\left(\mathfrak{u}^{v}\right)^{2}\right) \exp (h) \\
& \hat{H}_{w x}=\frac{\beta_{3}}{3 \cos (2 k) \beta_{2}} \hat{H}_{w w} \\
& \hat{H}_{x x}=-\hat{H}_{w w}
\end{aligned}
$$

where

$$
\begin{aligned}
& \beta_{1}=-\partial_{w} \partial_{w} h+4 \partial_{x} k^{2}-\partial_{x} \partial_{x} h+4 \partial_{w} k^{2}+2 \partial_{w} \partial_{w} a+2 \partial_{x} \partial_{x} a \\
& \beta_{2}=2 \partial_{w} \partial_{x} k-2 \partial_{w} a \partial_{x} k+\partial_{w} h \partial_{x} k-2 \partial_{x} a \partial_{w} k+\partial_{w} k \partial_{x} h \\
& \beta_{3}=-3 \cos (2 k) \partial_{w} \partial_{w} k+3 \cos (2 k) \partial_{x} \partial_{x} k+3 \cos (2 k) \partial_{x} k \partial_{x} h \\
& \quad-6 \cos (2 k) \partial_{x} a \partial_{x} k+3 \cos (2 k)\left(2 \partial_{w} a-\partial_{w} h\right) \partial_{w} k-\frac{1}{2} \sin (2 k) \beta_{1} \\
& \beta_{4}=-\frac{1}{2}\left(\left(\hat{\mathfrak{u}}^{u}\right)^{2}-\left(\hat{\mathfrak{u}}^{v}\right)^{2}\right) \sin (2 k)-\cos (2 k) \hat{\mathfrak{u}}^{u} \hat{\mathfrak{u}}^{v} .
\end{aligned}
$$

It follows that, for purely magnetic spacetimes, $\beta_{4}=0$ implies

$$
\hat{\mathfrak{u}}_{+}^{u}=\hat{\mathfrak{u}}^{v} \tan (k)
$$

or

$$
\hat{\mathfrak{u}}_{-}^{u}=-\hat{\mathfrak{u}}^{v} \cot (k)
$$

on $\mathcal{P}_{v} \cup \mathcal{P}_{u}$. However, by (B.1)-(B.2), only (B.12) leads to a real 4-vector which takes the form (10). 


\section{References}

[1] Stephani H, Kramer D, MacCallum M, Hoenselaers C and Herlt E 2003 Exact solutions of Einstein's field equations Cambridge Monographs on Mathematical Physics 2nd edn ed H Stephani, D Kramer, M MacCallum, C Hoenselaers and E Herlt (Cambridge: Cambridge University Press)

[2] Lessner G 2008 The 'complex trick' in five-dimensional relativity Gen. Rel. Grav. $402177-84$ 10.1007/s10714008-0625-5

[3] McIntosh C B G and Hickman M S 1985 Complex relativity and real solutions: I. Introduction Gen. Rel. Grav. 17 111-32

[4] Hall G S, Hickman M S and McIntosh C B G 1985 Complex relativity and real solutions: II. Classification of complex bivectors and metric classes Gen. Rel. Grav. 17 475-91

[5] Hickman M S and McIntosh C B G 1986 Complex relativity and real solutions: III. Real type- $N$ solutions from complex $N \otimes N$ ones Gen. Rel. Grav. 18 107-36

[6] Hickman M S and McIntosh C B G 1986 Complex relativity and real solutions: IV. Perturbations of vacuum Kerr-Schild spaces Gen. Rel. Grav. 18 1275-90

[7] McIntosh C B G, Hickman M S and Lun A W-C 1988 Complex relativity and real solutions: V. The flat space background Gen. Rel. Grav. 20 647-57

[8] McIntosh C B G and Hickman M S 1988 Single Kerr-Schild metrics: a double view Gen. Rel. Grav. $20793-811$

[9] Plebański J F, García-Compeán H and García-Díaz A 1995 Real Einstein spaces constructed via linear superposition of complex gravitational fields: the concrete case of non-twisting type $\mathrm{N}$ solutions Class. Quantum Grav. 121093

[10] Chudecki A 2010 Conformal Killing vectors in nonexpanding $\mathcal{H} \mathcal{H}$-spaces with $\Lambda$ Class. Quantum Grav. 27205004

[11] Newman E T and Janis A I 1965 Note on the kerr spinning-particle metric J. Math. Phys. 6 915-7

[12] Talbot C J 1969 Newman-Penrose approach to twisting degenerate metrics Commun. Math. Phys. 13 45-61

[13] Drake S P and Szekeres P 2000 Uniqueness of the Newman-Janis algorithm in generating the Kerr-Newman metric Gen. Rel. Grav. 32 445-58

[14] D’Inverno R A 1992 Introducing Einstein's relativity IInt. Elektron. Rundsch. (New York: Oxford University Press) pp 250-2

[15] Newman E T, Couch E, Chinnapared K, Exton A, Prakash A and Torrence R 1965 Metric of a rotating, charged mass J. Math. Phys. 6 918-9

[16] Basey T C 1975 A note on the generation of twisting ' $D$ ' metrics from non-twisting ' $D$ ' metrics Z. Nat.forsch. A 301200 (http://adsabs.harvard.edu/abs/1975ZNatA..30.1200B)

[17] Demiański M 1972 New Kerr-like space-time Phys. Lett. A 42 157-9

[18] Drake S P and Turolla R 1997 The application of the Newman-Janis algorithm in obtaining interior solutions of the kerr metric Class. Quantum Grav. 14 1883-97

[19] Viaggiu S 2006 Interior kerr solutions with the Newman-Janis algorithm starting with static physically reasonable space-times Int. J. Modern Phys. D 15 1441-53

[20] Herrera L and Jiménez J 1982 The complexification of a nonrotating sphere: an extension of the Newman-Janis algorithm J. Math. Phys. 23 2339-45

[21] Ibohal N 2005 Rotating metrics admitting non-perfect fluids Gen. Rel. Grav. 37 19-51

[22] McIntosh C B G 1992 Real Kasner and related complex 'windmill' vacuum spacetime metrics Gen. Rel. Grav. 24 757-72

[23] Lewis T 1932 Some special solutions of the equations of axially symmetric gravitational fields Proc. R. Soc. Ser. A 136 176-92

[24] Ehlers J and Kundt W 1962 Gravitation: an Introduction to Current Research ed L Witten (New York: Wiley)

[25] Harvey A 1989 Complex transformation of the Kasner metric Gen. Rel. Grav. 21 1021-6

[26] Harvey A 1990 Will the real Kasner metric please stand up Gen. Rel. Grav. 22 1433-45

[27] Wainwright J 1981 Exact spatially inhomogeneous cosmologies J. Phys. A: Math. Gen. 14 1131-47

[28] Agnew A F and Goode S W 1994 The separable cosmologies with heat flow Class. Quantum Grav. 11 $1725-42$

[29] Wainwright J 1979 A classification scheme for non-rotating inhomogeneous cosmologies J. Phys. A: Math. Gen. 12 2015-29

[30] Wainwright J and Goode S W 1980 Some exact inhomogeneous cosmologies with equation of state $p=\gamma \mu$ Phys. Rev. D 22 1906-9

[31] Ruiz E and Senovilla J M M 1992 General class of inhomogeneous perfect-fluid solutions Phys. Rev. D 45 1995-2005

[32] Senovilla J M M and Vera R 1998 Cosmological models separable in non-comoving coordinates Class. Quantum Grav. 15 1737-58 
[33] Senovilla J M M and Vera R 2001 New family of inhomogeneous $\gamma$-law cosmologies: example of gravitational waves in a homogeneous $p=\varrho / 3$ background Phys. Rev. D 63084008

[34] Wylleman L and Van den Bergh N 2006 Complete classification of purely magnetic, non-rotating and nonaccelerating perfect fluids Phys. Rev. D. 74 084001-14

[35] Bali R, Banerjee R and Banerjee S K 2009 Some LRS bianchi type $V I_{0}$ cosmological models with special free gravitational fields Electron. J. Theor. Phys. 6 165-73

[36] MacCallum M A H 1998 Hypersurface-orthogonal generators of an orthogonally transitive G2I, topological identifications, and axially and cylindrically symmetric spacetimes Gen. Rel. Grav. 30 131-50

[37] Castejón-Amenedo J and MacCallum M 1990 On finding hypersurface orthogonal Killing fields Gen. Rel. Grav. 22 393-415

[38] Maartens R, Mason D P and Tsamparlis M 1986 Kinematic and dynamic properties of conformal killing vectors in anisotropic fluids J. Math. Phys. 27 2987-94

[39] Brans C H 1975 Some restrictions on algebraically general vacuum metrics J. Math. Phys. 16 1008-10

[40] Szekeres P 1965 The gravitational compass J. Math. Phys. 6 1387-91

[41] Barnes A 2003 Purely magnetic spacetimes Proc. 27th Spanish Relativity Meeting (Encuentros Relativistas Espanoles), Alicante, Spain (http://xxx.lanl.gov/abs/gr-qc/0401068)

[42] Arianrhod R and McIntosh C B G 1992 Principle null directions of Petrov type I Weyl spinors: geometry and symmetry Class. Quantum Grav. 9 1969-82

[43] Maartens R 1997 Linearization instability of gravity waves? Phys. Rev. D 55 463-7 